\title{
Efficacy and safety of single-trocar technique for minimally invasive surgery of the chest in the treatment of noncomplex pleural disease
}

\author{
Marcello Migliore, MD, PhD, FETCS
}

From the Section of General Thoracic Surgery, Department of Surgery, University of Catania, Catania, Italy.

This study was supported in part by a grant of Dottorato di Ricerca in "Pre- and Postoperative Pathophysiology in Surgery" of University of Catania and European Union.

Received for publication Nov 13, 2002; revisions requested Feb 7, 2003; revisions received Feb 21, 2003; accepted for publication Feb 26, 2003.

Address for reprints: Marcello Migliore, MD, PhD, FETCS, University of Catania, Department of Surgery, Section of General Thoracic Surgery, Via Passo Gravina 187, Catania 95124, Italy (E-mail: mmiglior@ unict.it).

J Thorac Cardiovasc Surg 2003;126: $1618-23$

Copyright $\odot 2003$ by The American Association for Thoracic Surgery

$0022-5223 / 2003 \$ 30.00+0$

doi:10.1016/S0022-5223(03)00592-0
Objective: To assess the efficacy and the safety of a single-trocar technique for minimally invasive surgery of the chest in the management of multiple noncomplex thoracic diseases, a prospective study was performed and the results are presented.

Methods: Between October 1998 and December 2001, 100 patients underwent video-assisted thoracic surgery through a single trocar. The patients were divided into 4 groups as follows: (1) benign, (2) malignant, (3) pleural effusion, and (4) empyema. The following data were analyzed: age, sex, forced vital capacity, forced expiratory volume in 1 second, percentage of the predicted forced expiratory volume in 1 second, type of anesthesia, anesthesia time, surgery time, intraoperative complications, morbidity, chest tube removal, hospital stay, and follow-up.

Results: The patient population consisted of 64 men and 36 women with a mean age of 62 years (range 31-92 years). General anesthesia was used in 53 patients (25 double-lumen and 28 single-lumen tube) and local anesthesia and sedation in 47 patients. Talc pleurodesis was performed in 55 patients. Mean operative time was 65 \pm 37 minutes, $48 \pm 18$ minutes for simple and $67 \pm 37$ minutes $(P=.004)$ for complex pleural effusion. Mean anesthesia time was $102 \pm 85$ minutes. Chest tubes were removed after $5 \pm 2$ days. Mean overall hospital stay was $6 \pm 3$ days, $5 \pm 2$ days for benign diseases, $7 \pm 3$ days for malignant diseases, and $8 \pm 3$ for empyema. Morbidity was present in 19 patients. Two patients had intraoperative bleeding; 1 required a mini-thoracotomy to control it. There was no hospital mortality. Three patients had wound infection, and no patient with malignant diseases had port site metastasis.

Conclusion: Video-assisted thoracic surgery through a single trocar is simple, effective, and beneficial for all patients in the diagnosis and treatment of noncomplex diseases of the chest. Furthermore, with this newest type of technologically advanced instrumentation it is possible to carry out simple intrathoracic procedures without using additional ports.

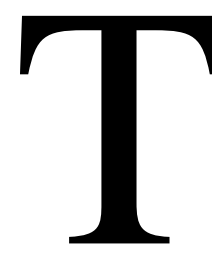

horacic surgeons are aware that video-assisted thoracic surgery (VATS) is not an operation but rather an approach to perform operations. The standard thoracoscope is the instrument most commonly used to perform diagnostic procedures in the pleural cavity; recently some minimally invasive techniques have improved the disadvantages of the classic thoracoscope..$^{1-4}$

Based on my institution's experience of using different ports available for VATS, an easy and reproducible minimally invasive technique was recently developed, ${ }^{4}$ which is defined as VATS through a single trocar (VATS-st). ${ }^{5}$ The technical advantages of the technique over the standard thoracoscopy or the use of videomediastinoscope have been reported. ${ }^{4}$ 
To assess the results and safety of VATS-st in the management of multiple noncomplex pathologies of the chest, I conducted a prospective study, the results of which are presented herein.

\section{Patients and Methods \\ Patients}

Between October 1, 1998, and August 31, 2001, 100 patients underwent VATS-st at the General Thoracic Surgery department at the University of Catania, in Catania, Italy. All patients had a thorough preoperative workup including medical history, chest radiography in 2 planes, respiratory function tests (RFTs), and electrocardiography. Thoracocentesis, examination with a flexible bronchoscope, computed tomographic scan of the chest and upper abdomen and brain, and bone scintigraphy were performed when appropriate.

The following parameters were analyzed: age, sex, forced vital capacity (FVC), forced expiratory volume in 1 second $\left(\mathrm{FEV}_{1}\right)$, percentage of the predicted forced expiratory volume in 1 second $\left(\% \mathrm{FEV}_{1}\right)$, type of anesthesia, anesthesia time, operative time, intraoperative complications, morbidity, chest tube removal, hospital stay, and follow-up.

To demonstrate the safety and efficacy of this technique in different diseases of the chest, the patients were divided into 4 groups as follows: (1) benign, (2) malignant, (3) pleural effusion, and (4) empyema.

The type of anesthesia and the use of a single- or double-lumen tube were chosen according to the general condition of the patient defined by the American Society of Anesthesiology (ASA) score and the pathology. Informed consent was obtained from each patient.

\section{Patient Selection and Definitions}

Patients were selected on the basis of a preliminary diagnosis of noncomplex thoracic disease. Noncomplex thoracic disease was defined as a disease of the chest that did not require an extensive dissection. Complex pleural effusion was used to identify an effusion characterized by multiple loculated pockets of fluid. Anesthesia time was the time interval between the entrance and the exit of the patient from the operating room. Surgery time was defined as the time interval between the skin incision and closure. The success rate of talc pleurodesis in pleural effusion was defined as absence of recurrence of pleural effusion within 30 days. The term "trapped lung" was used when pleural effusion was associated with thickness of the visceral pleura incarcerating the entire lung, and "trapped lobe" was used when the thick visceral pleura incarcerated only a lobe or segments of the lung.

\section{Operative Instrumentations}

The trocars commercially available for use are flexible (Flexipath, Ethicon, Inc, Somerville, NJ) and soft (Soft Thoracoport, Tyco International Ltd, Bermuda) with diameters ranging from 15 to 20 $\mathrm{mm}$. These characteristics allow the simultaneous introduction of the optic instrument and 1 or 2 standard 5 -mm thoracoscopic instruments. A $0^{\circ}$ or $45^{\circ}$ oblique viewing telescope of 5 or $10 \mathrm{~mm}$ can be used according to the disease. In this study, all types of 5-mm mini-invasive thoracoscopic instruments, including a com-

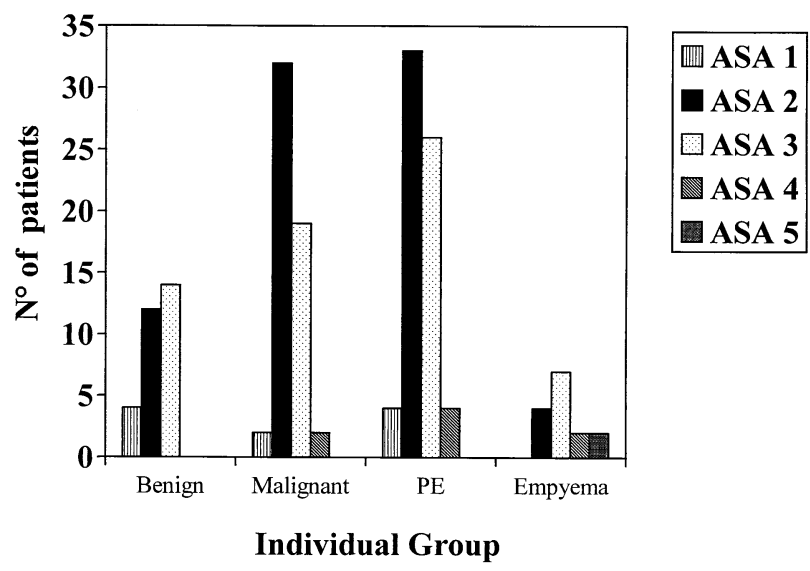

Figure 1. ASA status of the study group. PE, Pleural effusion.

bined suction and irrigating system, rotating scissors and graspers, 5-mm clip appliers, dissecting tampons, and ultrasound devices, were used. In some instances, standard open surgical instruments are useful.

\section{Anesthesia}

General anesthesia was chosen in 53 patients. Twenty-five patients had a double-lumen endotracheal tube to allow total collapse of the ipsilateral lung, and 28 patients had a standard endotracheal tube. Forty-seven patients had local anesthesia and sedation as previously described. ${ }^{6}$ Figure 1 reports the ASA scores.

\section{VATS-st Technique}

The surgical team included a primary surgeon, a first assistant, and a scrub nurse. The surgical technique was previously described. ${ }^{4}$ The patient was placed in the standard posterolateral decubitus position, but according to the specific location of the lesion and general condition, the operation was sometimes performed with the patient in the supine or anterolateral position. A 2-cm skin incision was performed in the planned intercostal space and the parietal pleura was opened under direct vision to 4 or $5 \mathrm{~cm}$. The index finger was used to lyse the possible adhesions of the lung to the chest wall, and the trocar was always inserted by means of a Kelly clamp. Once the trocar was positioned through the intercostal muscles, it became oval with the maximal diameter along the intercostal space; this structural modification was more evident on the pleural side. For improved flexibility and the ability to maneuver the instruments, a pyramid was created with a base in the pleura and the apex in the skin.

The chest drain was inserted under visual control. Postoperatively, chest radiographs were taken daily to ensure full lung expansion and to check for any pneumothorax or residual effusion. The chest drainage was under suction for at least 24 hours, and the tube was removed the day after the procedure or when the drainage was less than $100 \mathrm{~mL}$ daily.

\section{Statistical Analysis}

Demographic data are reported as mean \pm SD. We used the Student $t$ test for parametric results and the Mann-Whitney rank- 
TABLE 1. Preoperative diagnosis in the study population

\begin{tabular}{lc}
\hline & $\begin{array}{c}\text { No. of } \\
\text { patients }\end{array}$ \\
\hline Pleural effusion & 70 \\
Pleural thickening & 13 \\
Thoracic mass & 4 \\
Empyema & 5 \\
Mediastinal mass & 2 \\
Hydro-pneumothorax & 2 \\
Large mediastinal mass + pleural effusion & 1 \\
Hemothorax & 3 \\
Total & 100 \\
\hline
\end{tabular}

sum test and Kruskal-Wallis tests for nonparametric comparisons, while ordinal data were compared using the $\chi^{2}$ test.

\section{Results}

\section{Patients Demographics}

There were 64 male and 36 female patients with a mean age of $62 \pm 13$ years (range 31-92 years). The indications for the procedure in the 100 patients are reported in Table 1. Respiratory function tests showed an FVC of $2.2 \pm 0.83$ (range 0.88-4.17) and an $\mathrm{FEV}_{1}$ of $1.55 \pm 0.64$ (range $0.38-3.35$ ); mean $\% \mathrm{FEV}_{1}$ was $66 \% \pm 20 \%$ (range $24 \%$ $124 \%$ ). Twenty patients had $\mathrm{FEV}_{1}$ less than $50 \%$. Undiagnosed pleural effusion after thoracocentesis was present in 16 patients. A known history of lung cancer was present in 16 patients, breast cancer in 9 patients, and thymoma, nonHodgkin lymphoma, laryngeal cancer, left kidney cancer, bladder cancer in 1 patient each.

\section{Outcome of the Operations}

There were 2 instances of intraoperative bleeding (2\%). One patient required a $5-\mathrm{cm}$ mini-thoracotomy and the other a simple compression. In 4 cases (4\%) it was necessary to insert another trocar. During some phases of the operation, 2 surgical instruments were always used simultaneously with the optic. Trapped lobe was present in 9 and trapped lung in 4 patients. Mean operative time was $65 \pm 37$ minutes (range 20-195 minutes) and mean anesthesia time was $102 \pm 85$ minutes. Chest tubes were removed after $5 \pm$ 2 days (range 2-14 days). Hospital stay was $6 \pm 3$ days (range 2-15 days). Histologic studies was conducted in 95 patients as shown in Table 2. In 5 patients pleural biopsy was not performed because no macroscopic lesions were present. Morbidity was present in 19 patients (19\%). Atrial fibrillation, air leak, and hyperpyrexia were noted to be related to morbidity. Three patients had wound infection and no patients with malignant diseases had port site metastasis. There was no hospital mortality. Follow-up was complete with a mean of $11 \pm 8$ months.
TABLE 2. Final diagnosis in the study population

\begin{tabular}{lr}
\hline Benign disease & $\mathbf{4 0}$ \\
\hline Empyema & 15 \\
Chronic pleurisy & 10 \\
Fibrous tumor of the pleura & 9 \\
Hemothorax & 3 \\
Lipoma & 1 \\
Cavernous hemangioma & 1 \\
Tuberculosis & 1 \\
Malignant disease & 55 \\
Lung cancer & 24 \\
Adenocarcinoma & 16 \\
Squamous cell & 6 \\
Small cell & 2 \\
Metastatic cancer & 16 \\
Breast & 9 \\
Thymus & 2 \\
Bladder & 2 \\
Rhabdomyosarcoma & 1 \\
Kidney & 1 \\
Larynx & 1 \\
Mesothelioma & 13 \\
Malignant lymphoma & 2 \\
Idiopathic & 5
\end{tabular}

\section{Benign Diseases}

There were 30 patients with benign disease ( 20 men, 10 women) with a mean age of 60 years (range 43-89 years). All patients with empyema were excluded. Hepatic cirrhosis was present in 5 patients. Three patients had clotted hemothorax. Surgery time was $57 \pm 39$ minutes (range 20-180 minutes) and anesthesia time was $95 \pm 66$ minutes (range 30-275 minute). Mean time of chest tube removal was $4 \pm$ 2 days (range 2-11 days) and hospital stay was $5 \pm 2$ days (range 2-12 days). There were no intraoperative complications and morbidity was $8 \%$. Talc pleurodesis was performed in 4 patients older than 80 years.

\section{Malignant Diseases}

There were 55 patients with malignant disease ( 33 men, 22 women) with a mean age of $64 \pm 11$ years (range 32-89 years). One patient had a large mediastinal mass simultaneously with a left pleural effusion (Figure 2). Surgery time was $59 \pm 27$ minutes (range 20-140 minutes) and anesthesia time was $102 \pm 45$ minutes (range 30-225 minutes). In 4 patients with trapped lung an indwelling pleural catheter was inserted. Chest tubes were removed at $5 \pm 2$ days (range 2-9 days) and hospital stay was $6 \pm 2$ days (range 2-10). Morbidity was present in 12 (22\%) patients (atrial fibrillation and hyperpyrexia). Talc pleurodesis was performed in 51 patients with a success rate of 90\% (46 patients).

\section{Pleural Effusion}

There were 67 patients with pleural effusion: 34 of them had simple effusion (19 men and 15 women) with a mean age of 
$62 \pm 12$ years and 33 patients had complex effusion (19 men and 14 women) (Figure 3, $A$ and $B$ ) with a mean age of $61 \pm 15$ years. Surgery time was $48 \pm 18$ minutes for simple and $67 \pm 37$ minutes for complex effusion $(P=$ $.004)$. Anesthesia time was $83 \pm 37$ and $115 \pm 55$ minutes, respectively $(P=.005)$. Chest tubes were removed at $5.1 \pm$ 1.8 for simple and $4.8 \pm 2.4$ for complex pleural effusion, and hospital stay was $7 \pm 3$ versus $6 \pm 3$ days. Pleurodesis was performed in 29 patients with simple and 11 patients with complex pleural effusion.

\section{Empyema}

There were 15 patients (10 men, 5 women) with a final diagnosis of stage II empyema with a mean age of $65 \pm 15$ years (range 16-92 years). Eight patients underwent surgery under local anesthesia and sedation and 7 patients under general anesthesia ( 2 patients with a single- and 4 patients with a double-lumen tube). The flexible trocar was placed through the latissimus dorsi in 3 patients. One patient had a "simple" pleural empyema after pneumonectomy. One patient with empyema, following repeated thoracocentesis, had a mesothelioma (Figure 3, $A$ and $B$ ). One patient had an empyema complicating a hemothorax. In 2 patients "decortication" of a lobe was performed. In 2 cases it was necessary to insert another trocar. One patient had a prolonged air leak ( $>7$ days). Surgery time was $75.7 \pm 18.8$ minutes. Anesthesia time was $115 \pm 72.3$ minutes. Chest tubes were removed at $7.4 \pm 3.7$ days and hospital stay was $7.8 \pm 3.1$ days. No patient had recurrence of empyema.

\section{Discussion}

In the past decade, VATS gained popularity because of its minimally invasive technique. Rusch and Mountain $^{1}$ described the advantages of a mediastinoscope to diagnose and treat pleural diseases. Ozuner and colleagues ${ }^{2}$ used the mediastinoscope to perform a pericardial window to treat a recurrent pericarditis. Wittmoser ${ }^{3}$ used a single cannula to performed sympathectomy and vagotomy, and recently my group ${ }^{4}$ described the advantage of VATS-st to diagnose and treat a wide range of thoracic diseases. In some circumstances the use of VATS-st avoids multiple skin incisions for positioning of trocars, thus decreasing the risk of intercostal nerve damage and postoperative chest pain.

Several factors that can influence the outcome of a VATS-st technique operation. First, the indications for the operation must fit in a "noncomplex thoracic diseases" classification. Second, the trocar must be flexible. Third, the location where the trocar is inserted must be appropriate (based on chest radiographs and computed tomographic scan). The strategic position of the trocar will lead to a successful operation. Although the flexible port was placed posteriorly in 3 patients with empyema, I do not advocate the insertion of the trocar through the latissimus dorsi be-

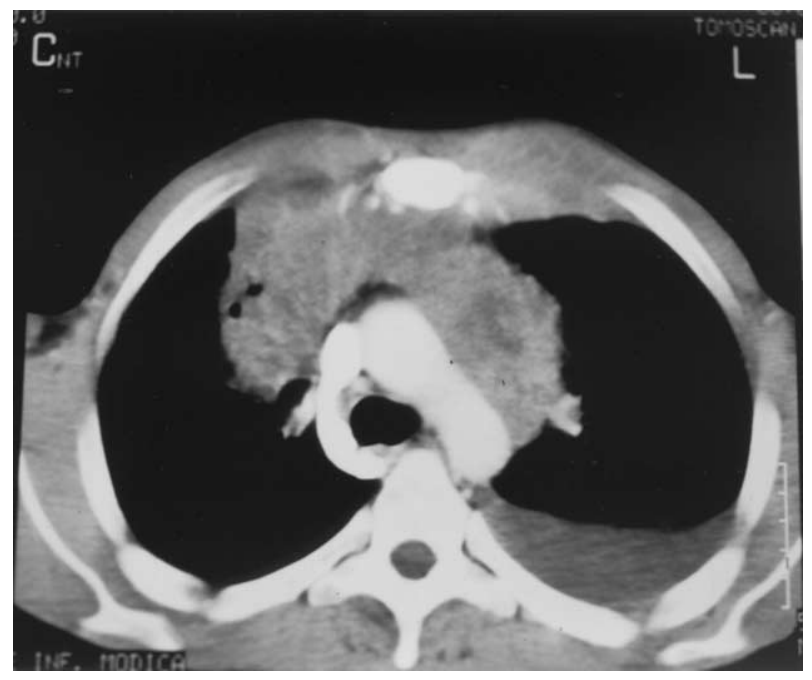

Figure 2. A large mass in the anterior mediastinum is associated with left pleural effusion. Biopsies of the mass and the simultaneous treatment of the effusion with talc were performed.

cause the intercostal space is smaller, and the chest tube also has problems of kinking and causing discomfort.

In pleural effusion cases RFTs should be performed after thoracocentesis. Nevertheless, when the operation is performed with the patient under local anesthesia and sedation, I do not recommend evacuating the fluid before the operation. I agree with Rusch and Mountain that in cases of pleural effusion, patients can tolerate the procedure under local anesthesia and sedation extremely well, because these patients are accustomed to living their life with a partially collapsed lung. ${ }^{1}$ It is also advisable to perform pleurodesis without a definitive diagnosis in patients older than 80 years with recurrent pleural effusion. This approach was used in 4 patients. Simple and loculated pleural effusion required different operative and anesthesia times $(P=.004)$, demonstrating the complexity of the disease and leading to an appropriate type of anesthesia and surgery. Malignant pleural effusion is best treated by thoracoscopic techniques. ${ }^{7,8} \mathrm{I}$ generally prefer talc pleurodesis instead of a long-term dwelling pleural catheter ${ }^{9}$ because the latter leads to patient discomfort due to daily maintenance. By using VATS-st, it is easy to grasp the tip of the cannula of the talc spreader to guide stratification of the talc on the "blind" surface of the lung. The posterior and mediastinal surfaces of the lobes can be exposed via a 5-mm endoscopic tampon. Talc is routinely administered in patients with trapped lobe to permit the adhesion between the parietal and visceral pleura of the remaining lung. The use of the pleuroperitoneal shunt or long-term indwelling pleural catheter is reserved for those patients with a trapped lung. ${ }^{9-11}$

Malignant pericardial effusion is an indication for VATS-st, except in patients with a previous pneumonec- 

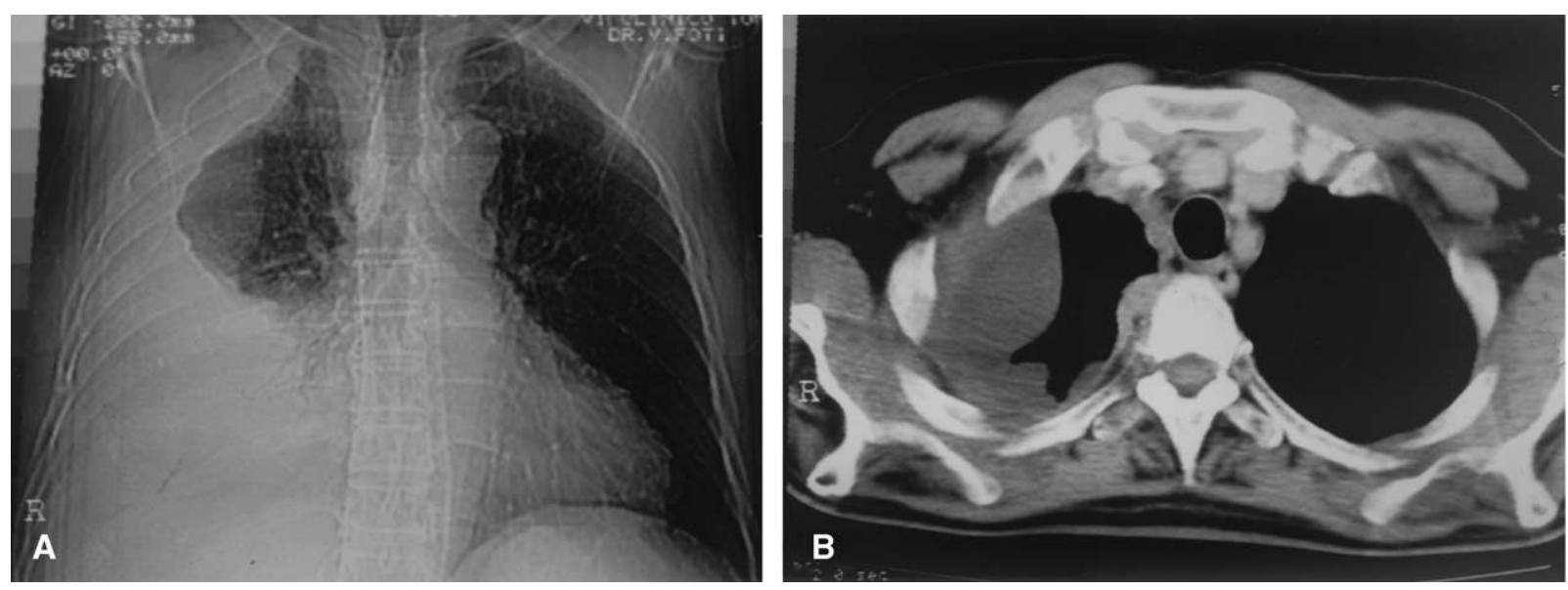

Figure 3. Preoperative chest radiograph (A) and computed tomographic scan (B) of an empyema after repeated thoracocentesis in a patient with mesothelioma.

tomy in whom a pericardioperitoneal shunt would be the preferred technique. $^{12}$

Mediastinal masses need a selective approach for diagnosis. ${ }^{13}$ Recently, it has been demonstrated that a thoracoscopic approach is indicated in some instances. ${ }^{14,15} \mathrm{I}$ do not advocate the transpleural biopsy of mediastinal masses because of the risk of disseminating the tumor into the pleural cavity. Nevertheless, when a large undiagnosed mass of the anterior mediastinum (Figure 2) is associated with pleural effusion, VATS-st allows simultaneous biopsies of the mediastinal tumor and definitive treatment of the pleural effusion. I speculate that mediastinal mass and pleural effusion may be associated, and I consider the latter a metastatic expression of the mass. It was discovered that the parietal pleura was already invaded by the mediastinal tumor in these patients. Furthermore, catastrophic complications of difficult mediastinoscopies can be avoided. ${ }^{16}$

It has already been demonstrated that stage II empyema is well treated with thoracoscopy by disruption of the loculations and complete drainage of the infected space, ${ }^{17}$ as confirmed in my experience. Although empyema was diagnosed preoperatively in 5 patients, the final diagnosis of empyema was confirmed in 15 patients on the basis of intraoperative findings, pleural biopsies, and cultural analysis of the pleural fluid. In some circumstances, localized lung "decortication" can also be performed. As confirmed by Hollaus and colleagues, ${ }^{18}$ if a "simple" pleural empyema develops after pneumonectomy, the pleural cavity can be sterilized by a thoracoscopic approach, avoiding further invasive procedures. ${ }^{19,20}$

The following limitation of this study must be kept in mind: this trial is not a comparison of VATS-st and the standard port technique, and this creates the potential for criticism in technique selection. Further investigative work may be required to confirm the advantages and the potential shortcomings of the VATS-st over several 5-mm incisions.

In conclusion, VATS-st is simple, effective, and beneficial for all patients in the diagnosis and treatment of noncomplex diseases of the chest. Furthermore, with this newest type of technologically advanced instrumentation, it is possible to conduct simple intrathoracic procedures without using additional ports.

I acknowledge Kim Nguyen, PharmD, and Arnold Estrada, PharmD, for excellent assistance. I am also much obliged to the medical and nursing staff of the thoracic surgery unit at Tomaselli Hospital, Catania, Italy.

\section{References}

1. Rusch VW, Mountain C. Thoracoscopy under regional anesthesia for the diagnosis and management of pleural disease. Am J Surg. 1987; 154:274-8.

2. Ozuner G, Davidson PG, Isnberg JS, McGinn JT. Creation of a pericardial window using thoracoscopic techniques. Surg Gynecol Obstet. 1992;175:69-71.

3. Wittmoser R. Thoracoscopic sympathectomy and vagotomy. In: Cuschieri A, Buess G, Perissat J, editors. Operative manual of endoscopic surgery. New York: Springer-Verlag; 1992. p. 110-33.

4. Migliore M, Deodato G. A single-trocar technique for minimallyinvasive surgery of the chest. Surg Endosc. 2001;15:899-901.

5. Migliore M, Deodato G. Thoracoscopic surgery, videothoracoscopic surgery or VATS: a confusion in definition. Ann Thorac Surg. 2000; 69:1990-1.

6. Migliore M, Giuliano R, Aziz T, Saad R, Sgalambro F. Four step local anesthesia and sedation for thoracoscopic diagnosis and management of pleural diseases. Chest. 2002;121:2032-5.

7. LoCicero J. Thoracoscopic management of malignant pleural effusion. Ann Thorac Surg. 1993;56:641-3.

8. Danby CA, Adebonojo SA, Moritz DM. Video-assisted talc pleurodesis for malignant pleural effusion utilizing local anesthesia and IV sedation. Chest. 1998;113:739-42.

9. Putnam JB, Garrett GL, Swisher SG, et al. Outpatient management of malignant pleural effusion by a chronic indwelling pleural catheter. Ann Thorac Surg. 2000;69:369-75. 
10. Gene O, Petrou M, Ladas G, Goldstraw P. The long-term morbidity of pleuroperitoneal shunts in the management of recurrent malignant effusions. Eur J Cardiothorac Surg. 2000;18:143-6.

11. Little AG, Kadowaki MH, Ferguson MK, Staszek VM, Skinner DB. Pleuro-peritoneal shunting. Alternative therapy for pleural effusion. Ann Surg. 1988;208:443-50.

12. Molnar TF, Jeyasingham K. Pericardioperitoneal shunt for persistent pericardial effusions: a new drainage procedure. Ann Thorac Surg. 1992;54:569-70.

13. Ferguson MK, Lee E, Skinner DB, Little AG. Selective operative approach for diagnosis and treatment of anterior mediastinal masses. Ann Thorac Surg. 1987;44:583-6.

14. Rendina EA, Venuta F, De Giacomo T, et al. Comparative merits of thoracoscopy, mediastinoscopy, and mediastinotomy for mediastinal biopsy. Ann Thorac Surg. 1994;57:992-5.

15. Gossot D, Toledo L, Fritsc S, Celerier M. Mediastinoscopy vs thora- coscopy for mediastinal biopsy. Results of a prospective nonrandomized study. Chest. 1996;110:1328-31.

16. Schmidli J, Vogt PR, Genoni M, Turina MI. Surgical management of severe common carotid artery injury after mediastinoscopy. Ann Thorac Surg. 2001;72:2107-9.

17. Lackner RP, Hughes R, Anderson LA, Sammut PH, Thompson AB. Video-assisted evacuation of empyema is the preferred procedure management of pleural space infection. Am J Surg. 2000;179:2730.

18. Hollaus PH, Lax F, Wurnig PN, Janakiev D, Pridun NS. Videothoracoscopic debridement of the postpneumonectomy space in empyema. Eur J Cardiothorac Surg. 1999;16:183-6.

19. Clagett OT. Changing aspects of the etiology and treatment of pleural empyema. Surg Clin North Am. 1973;53:863-6.

20. Peppas G, Molnar TF, Jeyasingham K, Kirk AB. Thoracoplasty in the context of current surgical practice. Ann Thorac Surg. 1993;56:903-9. 